\title{
Prediction of Entrained Mass Flows through Vertical Openings in a Multiroom Fire Model
}

\author{
XAVIER EMILE BODART and MICHEL ROGER CURTAT \\ Groupe Recherche Service Feu \\ Centre Scientifique et Technique du Bâtiment \\ 84, Avenue Jean Jaurès \\ Champs-sur-Marne 77420, France
}

ABSTRACT

CIFI (Circulation dans un Immeuble des Fumées de l'incendie) is a computer program based on a multiroom zone model. It has been developed at CSTB for the purpose of predicting air and smoke movement in a multistorey building. In the previons versions of CIFI, the mass flows circulating from room to room through vertical openings were calculated from the differences in vertical pressure fields across these openings, but no estimate of entrainments was made. We introduced recently in CIFI a simple set of criteria in order to decide on the possibility of distributing each flow through a vertical opening between the two gaseous layers located downstream. This results in plumes and entrained flows into these plumes, which were approximately estimated. A description of our approach in given and applied to the interpretation of experiments at NBS.

KEYWORDS: Multiroom fire - Fire modelling - Fire safety engineeringSmoke movement.

\section{INTRODUCTION}

The Fire Research Group of CSTB has been working for several years on modeling of building fires. Special attention was given to the prediction of air and smoke movement in the whole building, which is an important practical interest. In the last two years, several improvements were introduced in our models for the physical description of phenomena, numerical resolution and graphic output. Most of them were incorporated into the CIFI model + program. The main features of CIFI mode1+computer program are described at Ref. 1., where an example of application is given.

CALCULATION OF GASEOUS MASS FLOW RATES THROUGH AND NEAR VERTICAL OPENINGS

As recalled by Emmons' lecture at the lst Symposium on Fire Safety Science (4), the flow through a vertical vent from a fire room to another room - and vice versa - has received considerable attention, but up to now there is no theory as a guide on what to do when one has to describe a complex flow pattern through vents in a zone model. We know that the technique commonly used for such a purpose just consists of integrations over opening heights of functions of static pressure difference fields across these openings (2). 
This kind of calculation leads to good estimates of mass and heat transport through vertical openings so long as the situation remains simple, e.g. an open door or window between a fire room and a quiescent infinite environment of uniform temperature. Moreover, in such a case the validity of simple techniques used for flow determination can be directly checked by means of appropriate tests. The general concept of a neutral plane within a vertical opening can evidently be criticized, but when a fire model predicts the location of a single neutral plane within an opening between a fire room and the outside, this neutral planedelineates a separation of an inflow from an outflow, and one is sure that both of these flows do exist in real world because the fire source is a heat pump which needs to be fed with the oxygen in a fresh air incoming flow, and then blows away a mixture of air and combustion products thus forming an outgoing flow.

In the case of a vertical opening between two rooms of a building, a pattern with six different flows theoretically hounded by the opening soffit and sill, two interfaces (one on each side of the opening) and three neutral planes can be encountered. This pattern, although much more complex than those of "simple" cases (usually a maximum of three flows and one neutral plane for a room opening on the outside), results from the mere application of the rough technique described above, to the case where there are two gaseous layers on each side of the opening, each of these layers being at uniform temperature. When facing this case of six flows and three neutral planes in a given opening, as considered above, (see bottom of Fig. 1), one begins to doubt the representativeness of the description, and to think of field models as much more suitable for the description of what is really going on.

Nevertheless, in order to remain within the framework of zone modeling, we previously decided to keep that rough flow determination technique in our models, and to develop an algorithm (2) capable of performing this task as fast as possible. Then, each calculated flow was assumed to mix with the gaseous zone located just downstream of it, except for flows leaving an upper layer which where asked to contribute exclusively to the creation the upper layer on the opposite side of the opening. This appeared to be rather unsatisfactory for several reasons :

- In the case of a fire room with an open window on a windward side, the upper layer of the room grew in size excessively, as nothing was implemented in order to send at least a fraction of the fresh air inflow reinforced by the wind, to the botton of the room.

- An inflow coming from an upper layer is not necessarily very hot : if just warm, why not $\mathrm{mix}$ it with gases of lower layer rather than sending it to upper layer?

- On the otherhand, when the above-mentioned flow is much hotter than gases dowstream, it creates a plume which entrains a certain amount of gas from the lower layer to the upper layer above; the entrainment flow rates can be important, especially when the entraining flow emerges into a room with a high ceiling.

These few considerations helped us to define a way for improvement of calculation of flows through and near vertical openings :

1. It is desirable to quantify gaseous entrainment into plumes createdby flows dowstream of openings, and the need is al1 the more urgent as the entrainment height is large. 

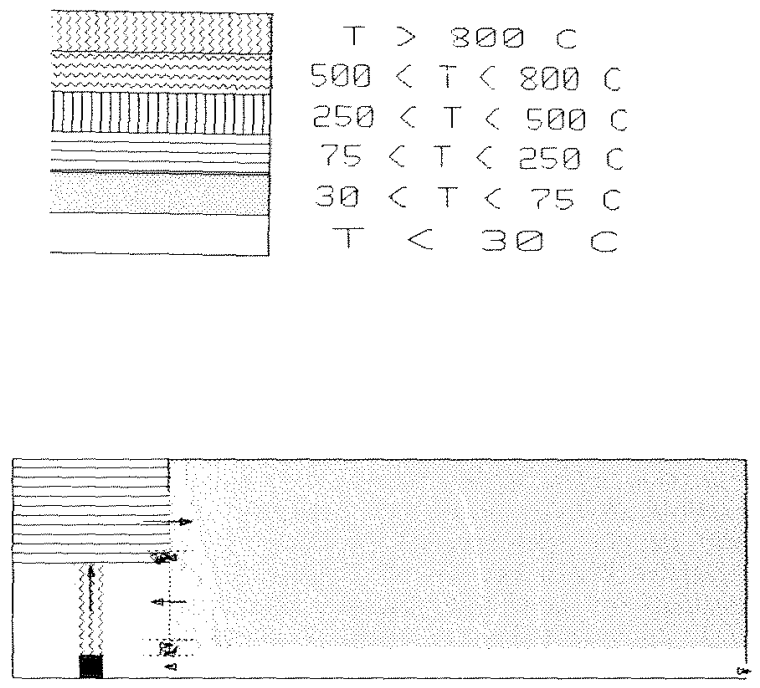

BURN ROOM CORRIDOR

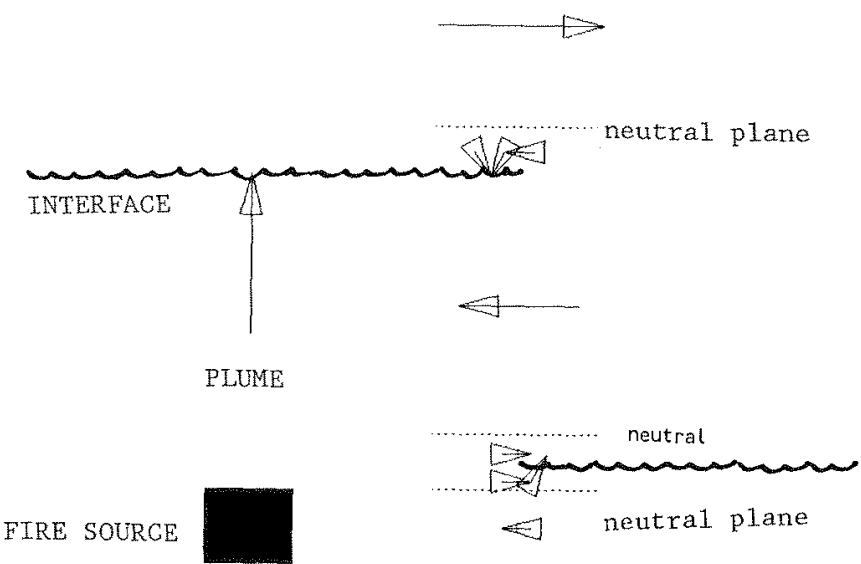

Figure 1 Example of CIFI graphic output. 
2. We wish to introduce a physically founded criterion instead of a merely logical criterion, in order to determine the destination of a flow through an opening. This destination being not necessarily unique, the flow may eventually be split into two flows, one of these going to the upper layer, the second one going to the lower layer of the room downstream.

3. We want a technique capable of performing the above-mentioned tasks in a self-consistent manner avolding discontinulties in mathematical formulations in order not to jeopardize the numerical "robustness" of numerical algorithms being used.

4. A fastalgorithm is preferred to a slow one !

In agreement with Nakamura \& Tanaka's considerations (5), saytng that the entrainment into door jet plumes must be correctly estimated principally when the entrainment height is large, and taking intoaccount that there is anyway a lack of theory concerning what is really going on in the vicinitry of the opening, we followed their decision to use the formulae for the far field region of Zukoski's fire plume model for computing the flow rate of door jet plumes. Besides, as we assume these plumes to be buoyant, we extended these formulae to the case of downvard plumes caused by cold Inflows, thus entraining upper layer gases to lower regions.

In order to calculate entraimment rates, Tanaka locates the position of a fictitious point heat source by equating the inflow rate through the opening (already determined at this step of evaluation) with the plume flow rate expressed as a function of the distance between this point source and another point (P) located somewhere in the incoming flow, within the opening : TANAKA considered (P) to be located half-way between the lower bound and the upper bound of the flow (6). Considering the case of a hot flow likely to create an upward plume downstream, it is clear that positioning (P) exactly at the upper bound of the flow would eliminate any entrainment when the interface of the downstream room is located under the opening soffit, since this interface then necessarily forms the upper bound of the flow (is the upper bound of the flow were a neutral plane, the incoming flow would be colder than the layer downstream and thencould not create any upward plume). Conversely, positioning (P) exactly at the lower bound of such a flow would evidently maximize the entrainment rate. These considerations will be easily extended to the case of downward plumes, the pattern having then to be turned upside down.

In order to evaluate the sensitiveness of the flow pattern to the location of $(P)$, we fixed a parameter in such a way that the location of (P) between both flow bounds (P), on an adimensionnal diagram just depends on this parameter. In the following, CIFI-A predictions result from a choice of this parameter causing (P) to be located at upper bounds (resp. lower bounds) of all flows with entrainment upwards (resp. downards). CIFI-B simulations used another choice causing (P) to be positioned halfway between lower and upper bounds of all flows.

In a previous version of his multiroom model, Tanaka estimated door jet entrainments by using McCaffrey's flame \& plume model : we also implemented them in our model and found that it yielded entrainment rates roughly twice bigger than those calculated from Zukoski's model (the one used for prediction of results commented in this paper). This recalls a 
comment already made by Beyler (9), who remarked that mass flow rates of air entrained into fire plumes, determined on the basis of profile integration also vary by roughly a factor of two.

For the calculation of air entrainment into upward smoke plumes developing along open shafts, Marshall (7) suggested one should consider such plumes as "half-plumes", the other "half-plume" forming a "ghost" plume. Then, he proposed to perform a calculation in two steps : first, calculate an entrainment from an equivalent source flow twice as big as the actual opening flow (being in fact, in his configurations, a flow at the end of a corridor emerging into the shaft); second, divided the result by two ! We think that such a technique is certainly relevant for the case of plumes whose size in a spanwise direction is large, such as plumes likely to be encountered during fires in shopping malls, along vertical walls of atria, etc...

In order to split a flow into two flows, one mixing with upper layer of the room downstream, the second one going into lower layer, Mitler (8), following Emmons considerations, proposed to divide the flow in such a way that the mixing proportions were linear with density difference, or with temperature difference. We incorporated in our model slightly different formulations, based on "pivot values" which are halfsums of the denstties of gaseous layers, calculated in each room. Then, the following set of criteria was taken :

- An incoming flow whose density is equal to or lower than the density of the upper layer of the room downstream, generates a plume which entrains gases from the lower layer to the upper layer of that room, unless this incoming flow emerges directly into the upper layer, in which case there is no plume and no entrainment. When entrainment occurs, its rate is calculated as described above, and of course no fraction of the incoming flow goes to the lower layer.

- The same was done for an incoming flow whose density is equal to or higher than that of the lower layer (no entrainment or full entrainment when incoming flow emerges into upper layer).

- When the density of the incoming flow is equal to the "pivot values" of the room, this flow goes to the layer into which it emerges, with no entrainment.

- When the density of the incoming flow in somewhere between the "pivot value" and one of the two densities of the gases forming the layers, there is either no split (and no entrainment) if this incoming flow density is closer to that of the layer into which it emerges than to that of the other layer, or a split, and hence an entralnment. Split and entrainment ratios are expressed linearly as functions of density differences, in order to ensure a continuous junction with the three afore-mentioned limit cases.

Although somewhat empirical, this set of criteria allows for all possible entrainment configurations and is then easily implementable in a computer program. Furthermore, when there are two flows leaving a gaseous layer, both of them circulating through a vertical opening, the first one going towards upper layer of the downstream room while the second one goes towards lower layer of that room, and when the common density of these two flows is intermediate between the two layer densities in downstream room, the set of criteria proposed above ensures that 
paradoxal configurations in which both flows would lead to plumes, one oriented upwards and the other one downwards, then impinging on each other in the vicinity of horizontal interface separating the two layers of downstream room, will be avoided, since only one plume is "allowed" to develop.

\section{COMPARAISON OF MODEL PREDICTIONS TO EXPERIMENTAL DATA :}

The tests (from N.B.S.) are described at reference (3). We chose for this presentation a fire in one of two adjacent rooms separated by a door open at $1 / 4$ width $(0.26 \mathrm{~m})$. A diffusion flame was produced in the burn room by a methane gas burner operated at several levels of heat release. We present here results obtained with a constant heat release of $100 \mathrm{~kJ}$. The helght of the interface between the lower and upper layers, $z d$, was estimated from thermocouples and photometers outputs and from visual observation. The value attributed to the temperature of upper layer (T) was obtained from a change of 10,15 , and $20 \%$ from the temperature given by the uppermost thermocouple. We kept here the values for a $10 \%$ change (symbol - ), and for a $20 \%$ change (symbol + ), to represent the experimental temperature $T$ of the upper layer. The predictions from two multiroom zone models presented in reference (3) are reproduced here, 1.e. those of the Zukoski and Kubota model (symbol: - - - ), and the Tanaka model (symbol:.............). CIFI results ( $A$ or $B$, as specified on the figures) are represented by continuous lines. In our CIFI computations, we used for thermal properties the data we had in our files from the names of used materials, that may differ slightly from the actual thermal properties. An example of CIFI graphic output is given at fig. 1 .

The general impression one has when looking at figures $2-5$ is that the predictions from the three models give a fair-to-good agreement with the tests results. One has to keep in mind that the definition of the interface between the gaseous zones is a simplification of the real world in which vertical gradients can be more or less important and that the meaning of the experimentally estimated values of $\mathrm{zd}$ and $\mathrm{T}$ is dependent on the basic assumption of existence of zones, and on the way one can calculate these zone characteristics from a limited number of thermocouples or photometers. The agreement observed on the figures shows the limits of the precision one can hope for from the present multiroom zone mode1s. This level of agreement seems to us very encouraging if we do not forget that the computer time consumed for one CIFI simulation 1s, for these simple fire situations, about $5 \mathrm{~min}$ on a VAX 780 .

The comparison between the predictions of CIFI and the other two models cannot lead to the conclusion that one model is clearly better than the others. For this kind of fire situation, the CIFI and the TANAKA model seem maybe better for predicting the very beginning of the evolutions of $z d$ and $T$.

We represented on some figures the differences between the results from CIFI-A and CIFI-B, when these differences were visible. On the basis of this set of experimental and computed data, one cannot say that the difference of assumptions is decisive as to the quality of our predictlons. We have to extend the fleld of comparisons to other tests before giving a global evaluation of the possible advantages of CIFI-B on CIFIA or other models. 
I (K)

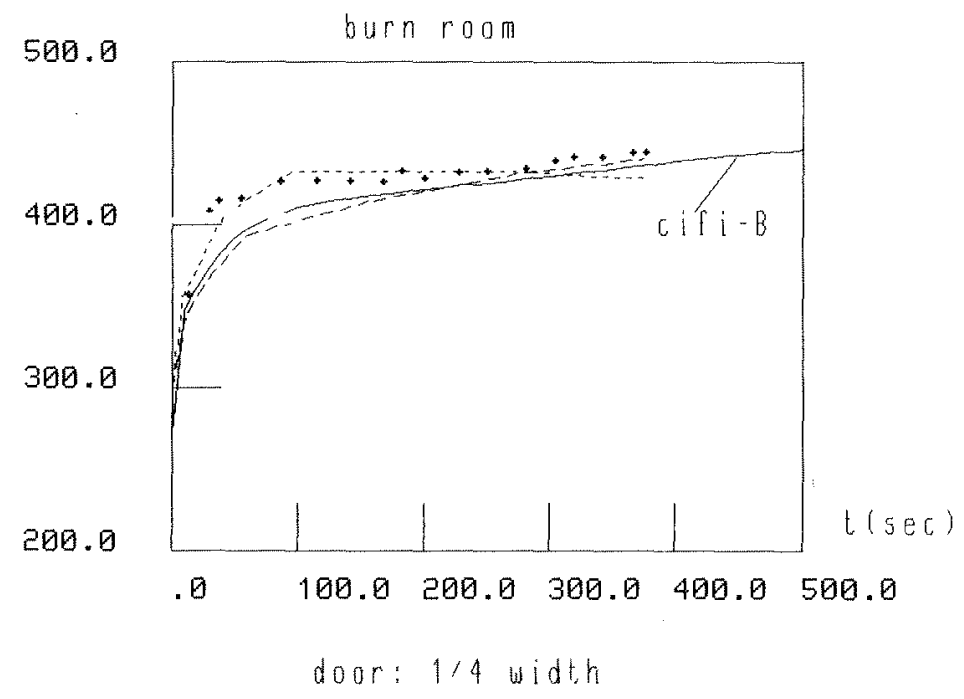

Figure 2

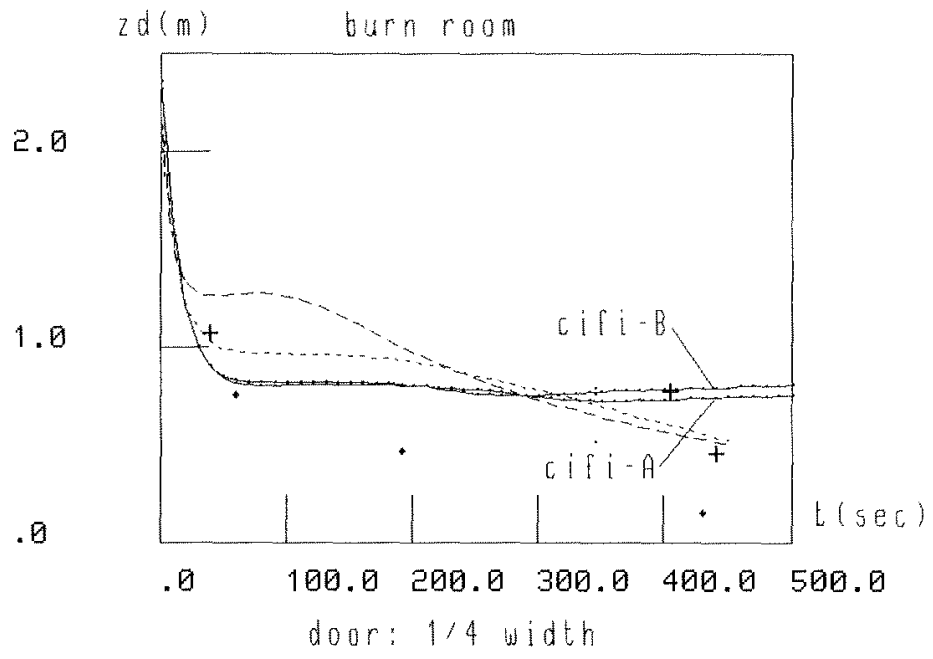

Figure 3 


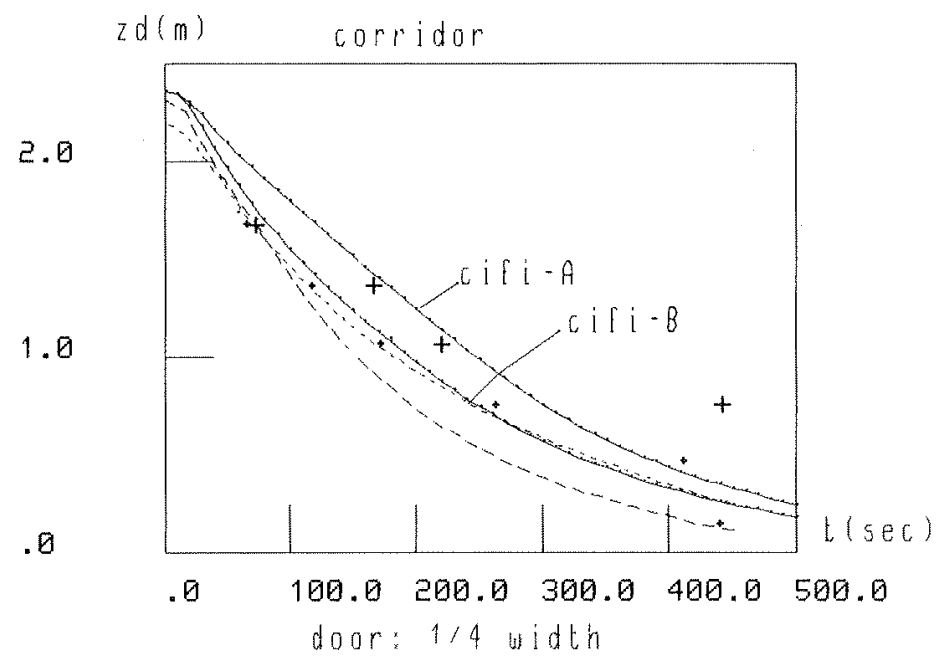

Figure 4

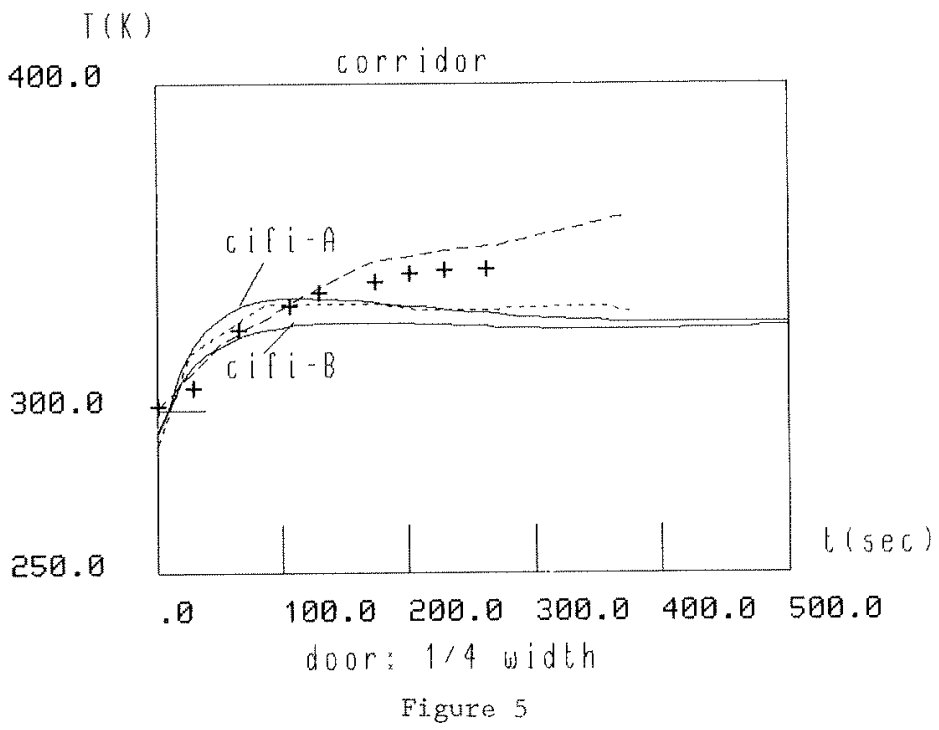




\section{CONCLUSTONS :}

Computed results from the CIFI model were compared to N.B.S. test data and to results computed from the Zukoski and Kubota model, and the Tanaka model. The most striking feature of this comparison is maybe that three models developed independently can give reasonable predictions, the differences between them being not much greater that the uncertainties on the quantitative definitions of the height of the interface and of the temperature of the upper layer.

This observation, plus the fact that the needed computational effort for user is small and computer time is short, are in favour of the use of multiroom zone models in engineering applications.

The formulation made in CIFI-B, towards a better description of mass and energy fluxes through a vertical opening between two rooms did not lead to an obvious increase of the predictive capabilities of our multiroom model, on the basis of a few tests. Important questions, which we could not answer definitely today, concern the orientation that has to be given now to the development of multi room fire models : is $1 t$ still useful to increase the complexity of zone models by trying to introduce better physical descriptions into them ? Are the available zone models good enough (and for which aspects of fire effects ?) for engineering applications to real world problems where the heat release has to be roughly estimated? For refinements should we not switch to the detalled description offered by field models ? Answers to these questions will obviously influence the degree of utilisation of models in Fire Safety. 
1. X. Bodart \& M. Curtat, "The Cifi Computer Code : Air and smoke movement during a fire in a building with ventilation ducts networks equipments", CIB W14 Wordshop, Berlin, May 1987.

2. X. Bodart, "Calcul des débits massiques échangés au travers d'une ouverture verticale", in rapport CSTB Programme F.I.S., October 1985.

3. W. Hones \& J. Quintiere, "Prediction of corridor smoke filling by zone models", Combustion Science \& Technology, Vol. 11, 1983.

4. H. Emmons' Lecture at the First International Symposium on Fire Safety Science, Gaithersburg (Md), October 1985.

5. T. Tanaka (Private communication, 1986).

6. T. Tanaka, "A Mode1 of Multiroom Fire Spread", NBSIR 84-2718, August 1983.

7. N.R. Marsha11, "Air Entrainment Into Smoke and Hot Gases in Open Shafts", Fire Safety Journal, 10, 1986.

8. H.E. MrTLER, "Zone Modelling of Forced Ventilation Fire", Combustion Science \& Technology, 39, 1984.

9. C.L. Beyler, "Fire Plumes And Ceiling Jets", Fire Safety Journal, vol. 11, nos $1 \& 2$, July/September, 1986. 